\title{
Broad Band Electromagnetic Characterization Method of Nematic Liquid Crystals Using a Coplanar Waveguide
}

\author{
J. Hinojosa \\ Departamento de Electrónica, Tecnología de Computadoras y Proyectos, Universidad Politécnica de Cartagena \\ C/. Doctor Fleming s/n, 30202 Cartagena, Spain
}

\begin{abstract}
A broad band electromagnetic extraction method of the dielectric tensor for nematic liquid crystals, associated to a measuring cell, is presented. The dielectric tensor is computed from $S$-parameter measurements obtained at the input planes of a coplanar waveguide supporting the nematic liquid crystal to be characterized, which is used as measuring cell. The extraction method of the dielectric tensor is based on the propagation of the dominant mode in the measuring cell. It is fast and easy. It uses analytical relationships, which are presented in this paper. The simulations results have demonstrated the validity of this extraction method in the $1 \mathrm{GHz}-40 \mathrm{GHz}$ frequency range for the electromagnetic characterization of nematic liquid crystals.
\end{abstract}

\section{INTRODUCTION}

In the last years, Frequency Agile Materials for Electronics (FAME) have been the aim of numerous studies to implement cheap electronics functions such as phase shifter, filter, etc. Among these materials, we can cite the nematic liquid crystals (NLCs) [1]. The development of microwave frequency agile devices based on NLCs requires knowing dielectric tensor properties in a broad band of frequency. These properties can be obtained from a characterization method using a measuring cell.

Experimental methods for measuring the parameters of anisotropic materials were investigated by several authors [2][4]. The measurements may be performed by means of the perturbation of cavity resonator, inside a rectangular waveguide, in free space, in a coaxial discontinuity or in stripline. Only Splingart et al. method [4] can be used to measure the dielectric tensor of NLCs in a broad band of frequency $(1 \mathrm{KHz}<f<10 \mathrm{GHz})$, since the others are limited in frequency or applicable to solid or particular materials [2]-[3].

Contrary to [4], which requires a rigorous electromagnetic analysis of the stripline cell from a numerical method as Spectral Domain Approach (SDA) [5], the technique presented here is easy and fast. Moreover, the measurements may be performed at frequencies above $10 \mathrm{GHz}$. It is based on a coplanar waveguide supporting the NLC to be characterized and used as measuring cell. The dielectric tensor is obtained from two $S$-parameter measurements of the measuring cell for two optical axis orientations of the NLC molecules: parallel and perpendicular to the RF electric field. The two orientations of the molecules are achieved by applying a bias voltage. $S$-parameters are performed at the input planes of the measuring cell from a network analyzer and on-wafer system covering a broad band of frequency $(1 \mathrm{GHz}-40 \mathrm{GHz})$. The dielectric tensor is extracted of those two $S$-parameters measurements using analytical relationships, which decrease considerably the computation time with respect to any numerical method. The measuring cell, the extraction method of the dielectric tensor based on analytical relationships and simulation results with isotropic and anisotropic materials under test are presented in this paper.

\section{MEASURING CELL}

The measuring cell (Fig. 1) is based on a coplanar waveguide supporting the NLC $\left(\hat{\varepsilon}_{r 2}\right)$ to be characterized. The coplanar waveguide is implemented on RT/Duroid 5880 substrate ( $\varepsilon_{r 1}=2.2-j 0.4 \times 10^{-3}$ at $10 \mathrm{GHz}$ ). The active part of the cell is obtained adding spacers all around the coplanar waveguide in order to make a cavity where the liquid crystal $\left(\hat{\varepsilon}_{r 2}\right)$ is deposited. The cavity thickness has the same thickness than RT/Duroid 5880 substrate: $h_{1}=h_{2}=787 \mu \mathrm{m}$. The coplanar structure was optimized in order to have characteristic impedance between $50 \Omega$ and $60 \Omega$ for the samples under test simulated in this paper: $W=260 \mu \mathrm{m}$ and $W+2 S=400 \mu \mathrm{m}$.

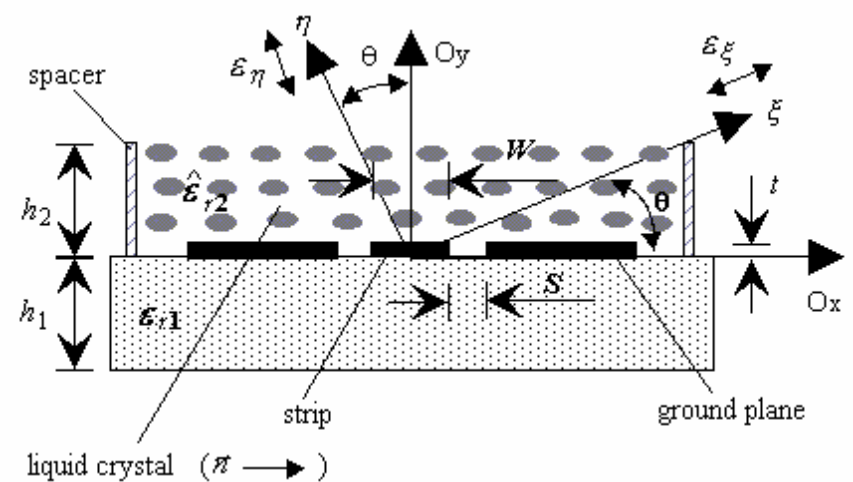

Fig.1. Cross section of the measuring cell.

A parallel $(\theta=0)$ or perpendicular $\left(\theta=90^{\circ}\right)$ orientation to the RF measuring electric field of nematic liquid crystal optical axis $(\vec{n})$ can be obtained inserting the measuring cell, respectively, between two vertical or horizontal plane electrodes (not shown in Fig. 1) and applying them a sufficient DC voltage. In this way, we can extract from two $S$-parameter 
measurements for two above respective orientations of the optical axis, the dielectric tensor $\left(\varepsilon_{\xi}=\varepsilon_{r 2 / /}\right.$ for $\theta=0$ and $\varepsilon_{\eta}=\varepsilon_{r 2 \perp}$ for $\theta=90^{\circ}$ ) of the NLC to be characterized.

\section{EXTRACTION METHOD OF THE DIELECTRIC TENSOR}

The extraction method is based on $S$-parameters measurements performed at the input planes of the measuring cell. The measurements of the $S$-parameters will be carried out by means of vector network analyzer (VNA) and on-wafer probing system as in [6]. This system connects the measuring cell with the VNA. The reference planes of the measurements at the access planes of the measuring cell are performed with an initial calibration. The permittivity tensor of the NLC under test is computed by resolving the reflection-transmission method [7] and by using an electromagnetic analysis method of the measuring cell. The reflection-transmission method requires the propagation to be dominant mode and its dispersion to be low in the measuring cell. The analysis method of the measuring cell is chosen and developed to be fast.

\section{Electromagnetic Analysis of the Cell}

The characteristic impedance of the measuring cell against frequency and several samples is plotted in Fig. 2 for the dominant mode. The characteristic impedance values were computed from commercially available finite element simulator with the above structure ( $W=260 \mu \mathrm{m}, W+2 S=400 \mu \mathrm{m}$, $h_{1}=h_{2}=787 \mu \mathrm{m}$ and $\left.\varepsilon_{r 1}=2.2-j 0\right)$. The intrinsic properties of the samples were considered constant in the $1 \mathrm{GHz}-40 \mathrm{GHz}$ frequency range. During the simulations, the conductors have been considered perfect $(t=0 \mu \mathrm{m}$ and $\sigma=\infty)$. As shown in Fig. 2, the dominant mode dispersion is lower than $1.5 \%$ in the frequency range covering $1 \mathrm{GHz}-40 \mathrm{GHz}$. Therefore, the reflection-transmission method can be used to extract the electromagnetic properties of the sample under test.

The dominant mode dispersion of the measuring cell being low, it is possible to substitute any numerical method by a quasi-static propagation analysis method of the measuring cell using analytical relationships derived from SchwarzChristoffel transformation (SCT) [8]. SCT allows to obtain the quasi-static effective permittivity and characteristic impedance for isotropic substrates $\left(\varepsilon_{r 1}, \varepsilon_{r 2}\right)$ :

$$
\begin{gathered}
\varepsilon_{r e f f}^{\prime}=\left(\varepsilon_{r 1}^{\prime} \frac{K\left(k_{1}\right)}{K\left(k_{1}^{\prime}\right)}+\varepsilon_{r 2}^{\prime} \frac{K\left(k_{2}\right)}{K\left(k_{2}^{\prime}\right)}\right)\left(\frac{K\left(k_{1}\right)}{K\left(k_{1}^{\prime}\right)}+\frac{K\left(k_{2}\right)}{K\left(k_{2}^{\prime}\right)}\right) \\
\left.Z_{c 0}=60 \pi / \sqrt{\varepsilon_{r e f f}} \cdot\left(\frac{K\left(k_{1}\right)}{K\left(k_{1}^{\prime}\right)}+\frac{K\left(k_{2}\right)}{K\left(k_{2}^{\prime}\right)}\right)\right]
\end{gathered}
$$

$k_{1}, k_{2}, k_{1}^{\prime}$ and $k_{2}^{\prime}$ are the bounds of the measuring cell (Fig. 1):

$$
k_{1}=\sinh \left(\frac{\pi W}{4 h_{1}}\right) / \sinh \left(\frac{\pi(W+2 S)}{4 h_{1}}\right) \text { and } k_{1}^{\prime}=\sqrt{1-k_{1}^{2}}
$$

$k_{2}$ and $k_{2}^{\prime}$ are obtained from (3) by replacing $h_{1}$ by $h_{2}$. $K\left(k_{1}\right), \quad K\left(k_{1}^{\prime}\right), \quad K\left(k_{2}\right)$ and $K\left(k_{2}^{\prime}\right)$ are the complete elliptical integrals of first order of modulus $k_{1}$ and $k_{2}$ and complementary modulus $k_{1}^{\prime}$ and $k_{2}^{\prime}$. These integrals are also computed from analytical relationships [9].

The effective loss tangent can be determined from loss factor as [10]:

$$
\operatorname{tgd}_{d e f f}=\frac{\varepsilon_{r e f f}^{\prime \prime}}{\varepsilon_{r e f f}^{\prime}}=q_{\operatorname{tgd} d 1} \cdot \operatorname{tgd} d_{d 1}+q_{\operatorname{tgd} d 2} \cdot \operatorname{tgd} d_{d 2}
$$

where

$$
\begin{aligned}
& q_{\operatorname{tgd} d 1}=\left(1-\varepsilon_{r 2}^{\prime} / \varepsilon_{r e f f}^{\prime}\right) /\left(1-\varepsilon_{r 2}^{\prime} / \varepsilon_{r 1}^{\prime}\right) \\
& q_{\operatorname{tgd} d 2}=\left(1-\varepsilon_{r 1}^{\prime} / \varepsilon_{r e f f}^{\prime}\right) /\left(1-\varepsilon_{r 1}^{\prime} / \varepsilon_{r 2}^{\prime}\right) \\
& \operatorname{tgd}_{d 1}=\varepsilon_{r 1}^{\prime \prime} / \varepsilon_{r 1}^{\prime} \text { and } \operatorname{tgd} d_{d 2}=\varepsilon_{r 2}^{\prime \prime} / \varepsilon_{r 2}^{\prime}
\end{aligned}
$$

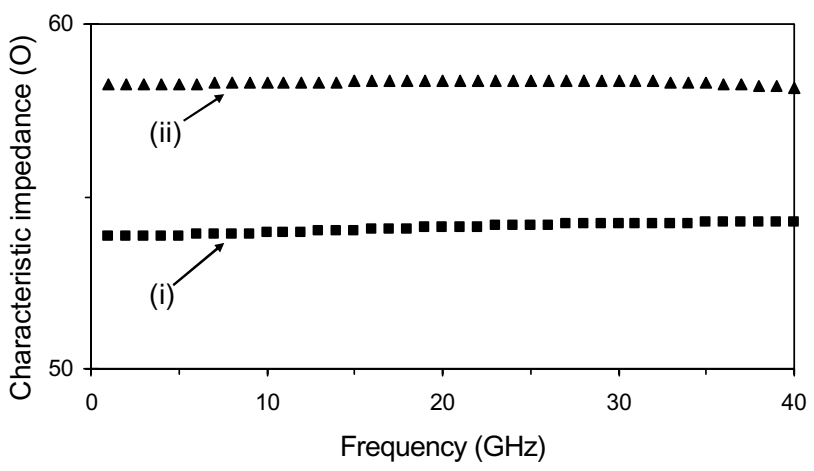

Fig. 2. Calculated characteristic impedances of the measuring cell against frequency and several materials under test. (i) $\varepsilon_{r 2}=5-j 0.25$. (ii) $\varepsilon_{\eta}^{\prime}=3, \varepsilon_{\xi}^{\prime}=5, \operatorname{tg} \delta_{\xi, \eta}=0$ and $\theta=0$.

\section{Resolution of the Reflection-Transmission Method}

The reflection-transmission method [7] allows the first reflection $(I)$ to be obtained at the input of the measuring cell and the first transmission $(T)$ along the measuring cell of length $d$. The resolution of the equations leads to the terms of complex effective permittivity and permeability $[7,11]$ : 


$$
\begin{gathered}
\varepsilon_{r e f f}=\varepsilon_{r e f f}^{\prime}-j \varepsilon_{r e f f}^{\prime \prime}=j \frac{c}{\omega d}\left(\frac{1-\Gamma}{1+\Gamma}\right)\left(\frac{Z_{c 0}^{\prime}}{Z_{0}}\right) \ln (T) \\
\mu_{r e f f}=\mu_{r e f f}^{\prime}-j \mu_{r e f f}^{\prime \prime}=-j \frac{c}{\omega d}\left(\frac{1+\Gamma}{1-\Gamma}\right)\left(\frac{Z_{0}}{Z_{c 0}^{\prime}}\right) \ln (T)
\end{gathered}
$$

where $c$ is the speed of light, $Z_{0}$ is the characteristic

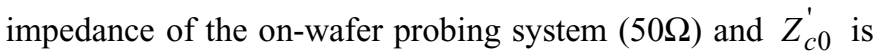
the characteristic impedance of the measuring cell when $\varepsilon_{r 2}=\mu_{r 2}=1$, which is obtained from (1) and (2).

However, for nonmagnetic materials to be measured, it is possible to increase the accuracy on dielectric characteristics by fixing the permeability at 1 in the reflection-transmission method. This leads us to look for only one complex unknown instead of two. Multiplying (8) and (9), we obtain [12][13]:

$$
\varepsilon_{\text {reff }}=-\left(\frac{j \ln T}{\omega d \sqrt{\varepsilon_{0} \mu_{0}}}\right)^{2}
$$

The exchange between complex effective permittivity and the sample desired relative permittivity is easily computed from (1) to (7).

\section{SIMULATION RESULTS}

In order to illustrate this extraction method, we have simulated with commercially available finite element simulator, the above configuration of the measuring cell with isotropic and anisotropic samples under test. The characteristics of these materials were considered constant in the $1 \mathrm{GHz}-40 \mathrm{GHz}$ frequency range. During the simulations, the conductors have been considered perfect $(t=0 \mu \mathrm{m}$ and $\sigma=\infty)$.

Fig. 3 corresponds to the simulation values for an isotropic sample: $\varepsilon_{r}^{\prime}=5$ and $\operatorname{tg} \delta=0.05$. The results correspond to those anticipated. The errors are less than $1 \%$ and $2.5 \%$ for $\varepsilon_{r}^{\prime}$ and $\varepsilon_{r}^{\prime \prime}$, respectively. This error is mainly due to the lack of precision of the above analytical relationships used in the extraction method.

Simulated values for an anisotropic sample $\left(\varepsilon_{\xi}^{\prime}=5, \varepsilon_{\eta}^{\prime}=4.7\right.$ and $\left.\operatorname{tg} \delta_{\xi, \eta}=0\right)$ are shown in Fig. 4. The results for the imaginary permittivity are not presented, since the obtained values are low (approximately $10^{-6}$ ). The results were obtained from two simulations. One gives the $S$-parameters for an optical axis of the molecule according to a parallel orientation $(\theta=0)$ to the RF measuring electric field and the other, the $S$ parameters for perpendicular orientation $\left(\theta=90^{\circ}\right)$. As it can be seen (Fig. 4), the propagation in the cell is slightly influenced by the anisotropic property of the sample under test [14]. The relative errors are less than $4 \%$ for $\varepsilon_{\xi}^{\prime}$ and $\varepsilon_{\eta}^{\prime}$ in the $1 \mathrm{GHz}$ $40 \mathrm{GHz}$ frequency range.

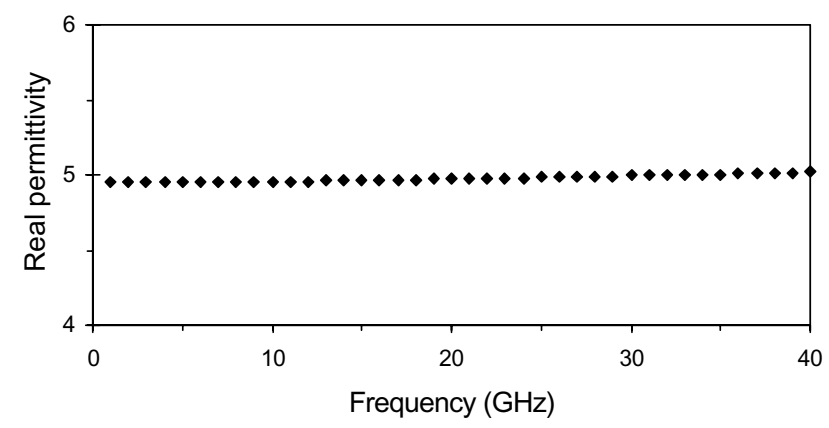

(a)

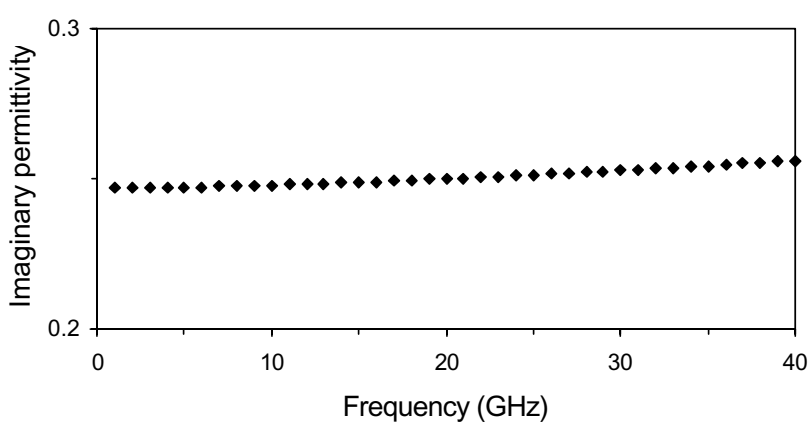

(b)

Fig. 3. Simulated data for an isotropic sample under test $\left(\varepsilon_{r 2}=5-j 0.25\right)$.

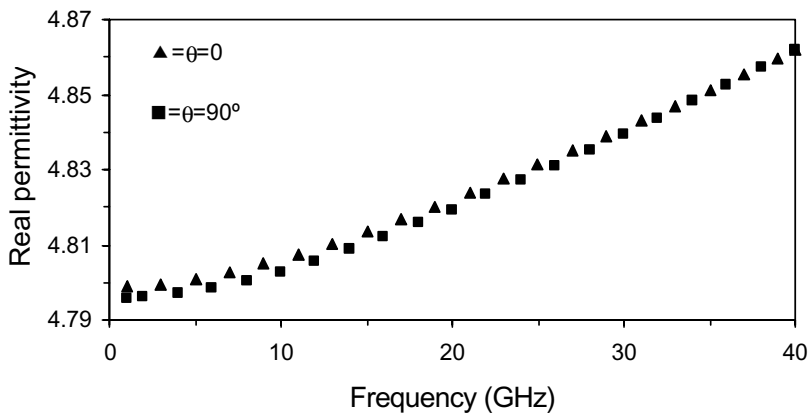

Fig. 4. Simulated data for an anisotropic sample under test $\left(\varepsilon_{\xi}=\varepsilon_{r 2 / /}=5\right.$ for $\theta=0, \quad \varepsilon_{\eta}=\varepsilon_{r 2 \perp}=4.7$ for $\theta=90^{\circ}, \operatorname{tg} \delta_{\xi, \eta}=0$ ) according to two orientations of the optical axis $\left(\boldsymbol{\Delta}=\varepsilon_{\xi}\right.$ for $\theta=0$ and $\varepsilon_{\eta}$ for $\left.\theta=90^{\circ}\right)$ 
Fig. 5 presents the relative error for relative permittivity extracted with this method versus the dielectric anisotropy $\left(\Delta \varepsilon=\varepsilon_{r / /}^{\prime}-\varepsilon_{r \perp}^{\prime}\right.$ with $\left.\varepsilon_{r 2 / /}^{\prime}=5\right)$ at $f=40 \mathrm{GHz}$. In the case of isotropic samples, the error is less than $1 \%$. However, this error increases for anisotropic samples, being less than $10 \%$ for a dielectric anisotropy below \pm 0.9 .

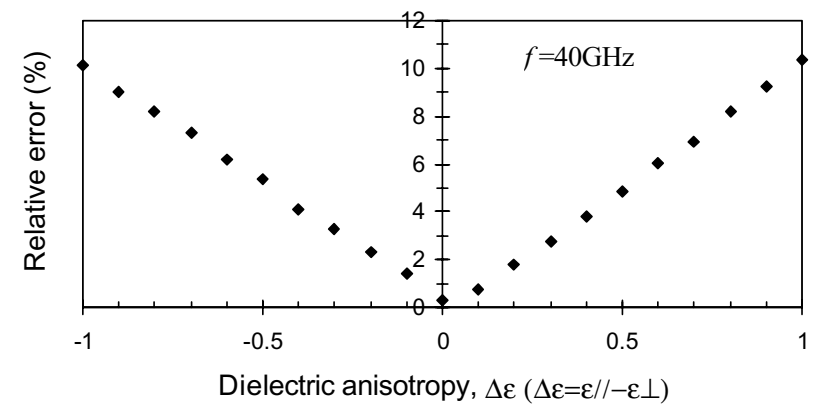

Fig. 5. Relative error for the extracted relative permittivity versus dielectric anisotropy at $f=40 \mathrm{GHz}$ with $\varepsilon_{r 2 / /}^{\prime}=5$.

\section{CONCLUSION}

A broad band extraction method of the dielectric tensor characteristics for nematic liquid crystals using a coplanar waveguide as measuring cell has been developed. The dielectric tensor properties are easily computed from a fast processing method of the $S$-parameters exploiting analytical relationships, which analyze the dominant mode in the measuring cell. The $S$-parameters are performed at the input planes of the measuring cell loaded with the nematic liquid crystal to be characterized. Simulated data for isotropic and anisotropic materials have demonstrated the validity of this method. This method should be suitable for experimental dielectric tensor electromagnetic characterizations of nematic liquid crystals with low dielectric anisotropy $(< \pm 0.9)$ in the $1 \mathrm{GHz}-40 \mathrm{GHz}$ frequency range.

\section{ACKNOWLEDGMENTS}

J. Hinojosa is grateful to Ministerio de Ciencia y Technología and Region de Murcia Fundación Séneca of Spain for the grants TIC2002-01266 and PB/39/FS/02, which have allowed the present research activity.

\section{REFERENCES}

[1] D. Dolfi, M. Labeyrie, P. Joffre and J. P. Huignard, "Liquid crystal microwave phase shifter," Electronics Letters, vol. 29, pp. 926-928, May 1993.
[2] J. P. Parneix, C. Legrand and S. Toutain, "Automatic permittivity measurements in a wide frequency range: Application to anisotropic fruid," IEEE Trans. Microwave Theory and Tech., vol. 82, pp. 2015-2017, Nov. 1982.

[3] N-E Belhadj-Tahar and A. Fourier-Lamer, "Broad-band simultaneous measurement of the complex permittivity tensor for uniaxial materials using a coaxial discontinuity," IEEE Trans. Microwave Theory and Tech., vol. 39, pp. 1718-1724, Oct. 1991.

[4] B. Splingart, N. Tentillier, F. Huret and C. Legrand, "Liquid crystals applications of RF and microwave tunable components," Molecular Crystal and Liquid Crystals, vol. 368 , pp. 183-190, 2001.

[5] T. Itoh and R. Mittra, "Spectral-domain approach for calculating the dispersion characteristics of microstrip lines," IEEE Trans. Microwave Theory and Tech., vol. 21, pp. 496499, July 1973.

[6] J. Hinojosa, K. Lmimouni, S. Lepilliet and G. Dambrine, "Very high broadband electromagnetic characterization method of film-shaped materials using coplanar," Microwave and Optical Technology letters, vol. 33, pp. 352-355, June 2002.

[7] W. B. Weir, "Automatic measurement of complex dielectric constant and permeability at microwave frequencies," Proceedings of the IEEE, vol. 62, pp. 33-36, January 1974.

[8] R. E. Collin, Field theory of guided waves, $2^{\text {nd }}$ Ed, IEEE Press, New York, 1976, pp. 263-273.

[9] -W. Hilberg, "From approximations to exact relations for characteristic impedances," IEEE Trans. Microwave Theory and Tech., vol. 17, pp. 259-265, May 1969.

[10] H. A. Wheeler, "Transmission-line properties of parallel strips separated by a dielectric sheet," IEEE Trans. Microwave Theory and Tech., vol. 13, pp. 172-185, March 1965.

[11] J. Hinojosa, "S-parameter broadband measurements oncoplanar and fast extraction of the substrate intrinsic properties", IEEE Microwave and Wireless Comp. letters, vol. 11, pp. 80-82, Feb. 2001.

[12] A. Boughriet, C. Legrand and A. Chapoton, "Non iterative stable transmission-reflection method for low-loss material complex permittivity determination", IEEE Trans. Microwave Theory and Tech., vol. 45, pp. 52-57, Jan. 1997.

[13] J. Hinojosa, K. Lmimouni and G. Dambrine, "Fast electromagnetic characterization method of thin planar materials using coplanar line up to V-band", Electronics Letters, vol. 38, pp. 373-374, April 2002.

[14] A-M. El-Sherbiny, "Hybrid mode analysis of microstrip lines on anisotropic substrates", In Proc. IEEE MTT-S, 1981, pp. 492-494. 